\title{
Nonlinear Observer for the turbulent wake of a square cylinder
}

\author{
Javeria Ahmed, Estelle Courtial, Pierre-Yves Passaggia, Matthieu Fruchard and Nicolas Mazellier
}

\begin{abstract}
Vortex shedding in the turbulent wake of a square cylinder is modeled by a minimal generalized empirical Galerkin model (GM). Pressure measurements on the sides and on the back of the bluff body are used as a system output to rebuild the system full state. Despite a large number of unresolved states and the intermittent character of the vortex shedding, the use of an observer makes possible the estimation of the shift mode and the growth rate of the dynamic model truncated at order two. A high gain observer is synthesized and both simulation and experimental results show promising applications for the feedback control of the vortex shedding in order to decrease the drag coefficient, even in the case of a turbulent wake flow at high Reynolds numbers.
\end{abstract}

\section{INTRODUCTION}

The analysis of wake flows and its impact on the energy consumption in the transportation sector becomes an increasingly important issue. Vehicles are often considered as bluff bodies where the flow is massively separated in the near wake. Predicting the flow inside the wake is a challenging issue and has a profound impact on our capability in controlling such flows. For instance in the case of vehicles at cruising speeds, a decrease of $2 \%$ in the drag coefficient can result in $1 \%$ energy consumption for road vehicles. This ratio becomes even closer to unity for naval and aerodynamic applications. However the estimation of the drag force on a vehicle remains a challenge [1]. Thus far, methods to determine the drag of a bluff body relies on dense measurements of pressure to ensure minimum errors for the calculation of the resulting forces exerted by the pressure and skin friction. In the case of bluff-body flows, the latter often plays an minor role and methods that can be used in real time for the observation of the drag coefficient from sparse pressure measurements and eventually the feedback control of such flows are of major scientific and industrial interest [2].

The main challenge in the mathematical/physical modeling and the prediction of wake dynamics resides in the number of states or modes necessary to describe the physics and how these modes contribute to drag force. Some of these modes seem to be necessary to accurately capture the broad range of unstable frequencies in a turbulent flow [3], [4], [5], [6]. These modes can be organized in a spectrum which counts several types of strongly amplified, self-excited, or intermittent modes which need to either be represented or filtered to capture and model the wake dynamics. Unless the

\footnotetext{
All the authors are with Laboratory PRISME, EA 4229, University of Orléans, INSA-CVL, 45072 Orléans, France. javeria.ahmed@etu.univ-orleans.fr, estelle.courtial, pierre-yves.passaggia, matthieu.fruchard, nicolas.mazellier @univ-orleans.fr
}

number of modes or states used to represent the dynamics is kept small, a dynamical system cannot be used in real time in a data-assimilation-type scheme where the sensors output can be used to predict drag in real time. In addition, the unstable character of the wake prevents the use of linear models which grow unbounded in time and therefore do not capture the finite amplitude dynamics [7], [8].

Recent methods have allowed for constructing nonlinear reduced order models solely based on sparse time-resolved measurements [9], [10]. In this framework, the wake flow behind a cylinder is a well documented flow where the primary instability is a Hopf-type bifurcation which, in the laminar regime, is known to reach a finite amplitude and saturate to a limit cycle. The saturation process was highlighted in [11], [12], [3], [13] by the interaction between the vortex shedding mode and the steady state, which induces a shift of the flow, also known as shift mode [14], [15]. While the vortex shedding mode is characterized by a growth rate and a frequency mode, the shift mode only possesses a decay rate which varies with the growth rate and both are strongly coupled [14], [15]. In the case of a cylinder, the shift mode is associated with a shortening recirculation region and is directly related to the magnitude of the vortex shedding, the latter being dependent on the Reynolds number. While this process is now well understood in the case of the laminar flow, this model scenario yet has to be tested for turbulent flow conditions at high Reynolds number. In particular, we are interested in the capacity of this empirical Galerkin model to capture the wake dynamics and the instantaneous evolution of the drag coefficient. In order to eventually implement a control law, it is of major importance to design an observer which can separate the turbulent dynamics from the vortexshedding mode and evaluate the pertinence of a simplistic dynamical model in representing the drag on a bluff body.

The present paper deals with a high gain observer synthesis in order to rebuild states that are not accessible to the measurement. We first provide a short mathematical description of the Galerkin model used for the observation. The observability of this empirical Galerkin model is then studied. However, for that kind of system, a natural feedback control objective is to bring the system back to a symmetric wake flow, corresponding precisely to the observability singularity of the system. We consequently show that a high gain observer synthesis using a dynamic extension and fake outputs may extend the proposed diffeomorphism image to the full state space. Simulations and experiments illustrate the efficiency and limitations of the proposed observer. 


\section{MODELING}

The evolution of fluid flow is governed by the well-known Navier-Stokes (NS) equations, a set of an incompressibility condition and a nonlinear partial differential equation. The NS equations are characterized by strong nonlinearities, high dimensionality and time-delays making fluid flow control a challenging area, especially if real applications are targeted. In the past fifteen years, reduced-order model (ROM) approaches have been extensively developed in the literature to cope with the aforementioned difficulties. The objective is to obtain a low-order ODE model from the NS equation. The most popular ways of obtaining ROMs are the Proper Orthogonal Decomposition (POD) [16], more recently the Dynamic Mode Decomposition [17], [18] and the Optimal Mode Decomposition [19]. Among ROMs, low-dimensional Galerkin models showed promising results for feedback control design [20].

In this study, a Galerkin model (GM) based on a KarhunenLoève expansion around the unstable steady NS solution $u_{s}$ is used. The flow $u(x, t)$ is then described by the orthonormal Galerkin approximation $u^{[N]}$ :

$$
u(x, t) \approx u^{[N]}=u_{s}+\sum_{i=1}^{N} a_{i}(t) u_{i}(x),
$$

where the velocity modes $u_{i}(x)$ depend on the location $x$ and the $a_{i}(t)$ describe the time evolution of the Fourier coefficients. The two first modes $(N=2)$ capture $96 \%$ of the perturbation energy. The shift-mode, noted $u_{\Delta}$, represents the energy exchange between the mean flow and the oscillatory perturbation. The Galerkin approximation of order three is then written as follows:

$$
u(x, t) \approx u_{s}+a_{1}(t) u_{1}(x)+a_{2}(t) u_{2}(x)+a_{\Delta}(t) u_{\Delta}(x) .
$$

The time evolution of the Fourier coefficients is described by [13]:

$$
\dot{a}(t)=F(a) \cdot a=\left(\begin{array}{ccc}
\mu & -1 & -a_{1} \\
1 & \mu & -a_{2} \\
a_{1} & a_{2} & -1
\end{array}\right) \cdot\left(\begin{array}{l}
a_{1} \\
a_{2} \\
a_{\Delta}
\end{array}\right)
$$

with $\mu>0$ the growth rate parameter. In cylindrical coordinates, setting the oscillation amplitude $A_{m p}=\sqrt{a_{1}^{2}+a_{2}^{2}}$, equations (3) can be rewritten :

$$
\left(\begin{array}{c}
\dot{A}_{m p}(t) \\
\dot{a}_{\Delta}(t)
\end{array}\right)=\left(\begin{array}{c}
\left(\mu-a_{\Delta}\right) A_{m p} \\
A_{m p}^{2}-a_{\Delta}
\end{array}\right) .
$$

In the sequel, we denote the state vector $x=\left(A_{m p} a_{\Delta} \mu\right)^{T} \in$ $\mathscr{X} \subset \mathbb{R}^{3}$ and consider the dynamic nonlinear system:

$$
\dot{x}=f(x)=\left(\begin{array}{c}
x_{1}\left(x_{3}-x_{2}\right) \\
x_{1}^{2}-x_{2} \\
0
\end{array}\right), y=h(x)=C x=x_{1},
$$

where $y$ is the measured output and $C=\left(\begin{array}{lll}1 & 0 & 0\end{array}\right)$.

\section{SYSTEM EQUILIBRIA AND OBSERVABILITY}

Theorem $1 \forall \mu \in \mathbb{R}_{+}^{*}$, system (5) equilibria are given by:

- $x^{*}=(00 \mu)^{T}$ is an unstable equilibrium ;

- $x^{* *}=(\sqrt{\mu} \mu \mu)^{T}$ is a locally stable equilibrium;

- $x^{* * *}=(-\sqrt{\mu} \mu \mu)^{T}$ is a locally stable equilibrium.

Proof: A first order analysis is sufficient to state on the stability of the equilibria. It is straightforward that $f(x)=0$ if and only if $x \in\left\{x^{*}, x^{* *}, x^{* * *}\right\}$ and we have

$$
\frac{\partial f}{\partial x}(x)=\left(\begin{array}{ccc}
x_{3}-x_{2} & -x_{1} & x_{1} \\
2 x_{1} & -1 & 0 \\
0 & 0 & 0
\end{array}\right)
$$

The eigenvalues of $\frac{\partial f}{\partial x}$ lie in the set

$$
\left\{0, \frac{\left(x_{3}-x_{2}-1\right) \pm \sqrt{\left(x_{3}-x_{2}\right)^{2}+2\left(x_{3}-x_{2}\right)+1-8 x_{1}^{2}}}{2}\right\} .
$$

The null eigenvalue is linked to the marginal stability of $x_{3}$, inherited from the last line of $\frac{\partial f}{\partial x}$ and simply expresses that $x_{3}$ is a constant.

- The eigenvalues of $\frac{\partial f}{\partial x}\left(x^{*}\right)$ lie in $\{0,-1, \mu\}$ so $x^{*}$ is a saddle point for $\mu>0$ and is an unstable equilibrium.

- The eigenvalues of $\frac{\partial f}{\partial x}\left(x^{* *}\right)$ lie in $\left\{0, \frac{-1 \pm \sqrt{1-8 \mu}}{2}\right\}$ so $x^{* *}$ is a stable equilibrium whose basin of attraction is $\{x \in$ $\left.\mathbb{R}_{+}^{*} \times \mathbb{R}^{2}\right\}$.

- The eigenvalues of $\frac{\partial f}{\partial x}\left(x^{* * *}\right)$ lie in $\left\{0, \frac{-1 \pm \sqrt{1-8 \mu}}{2}\right\}$ so $x^{* * *}$ is a stable equilibrium whose basin of attraction is $\left\{x \in \mathbb{R}_{-}^{*} \times \mathbb{R}^{2}\right\}$.

These local results can be checked using $V=(x-$ $\left.x_{e}\right)^{T} \frac{\partial f}{\partial x}\left(x_{e}\right)\left(x-x_{e}\right)$ as a Lyapunov candidate function for $x_{e} \in\left\{x^{*}, x^{* *}, x^{* * *}\right\}$.

Theorem 2 System (5) is observable on $\mathscr{S}=\left\{x \in \mathbb{R}^{3}\right.$ : $\left.\left|x_{1}\right|>\varepsilon\right\}$ for any $\varepsilon>0$.

Proof: Let the observation set $\mathscr{O}$ denote the smallest vector space that contains $h$ and closed under the Lie derivative $\mathscr{L}_{f}$, i.e. such that $\forall \sigma \in \mathscr{O}, \mathscr{L}_{\tau}(\sigma) \in \mathscr{O} . \mathrm{d} \mathscr{O}=$ $\operatorname{Span}\{\mathrm{d} \tau, \tau \in \mathscr{O}\}$ is the observability co-distribution where $\mathrm{d}$ denote the differential. A system is weakly observable if $\operatorname{dim} \mathrm{d} \mathscr{O}(x)=\operatorname{dim} \mathscr{X}$.

Using the output $y$ in (5), we compute the successive Lie derivatives

$$
\begin{aligned}
\mathscr{L}_{f}^{0} h(x) & =h(x)=x_{1} \\
\mathscr{L}_{f}^{1} h(x) & =x_{1}\left(x_{3}-x_{2}\right) \\
\mathscr{L}_{f}^{2} h(x) & =x_{1}\left[\left(x_{3}-x_{2}\right)^{2}-x_{1}^{2}+x_{2}\right] \\
\mathscr{L}_{f}^{3} h(x) & =x_{1}\left[\left(x_{3}-x_{2}\right)^{3}+\left(x_{3}-x_{2}\right)\left(3 x_{2}-5 x_{1}^{2}\right)+x_{1}^{2}-x_{2}\right]
\end{aligned}
$$

Differentiation at higher orders will still lead to expressions 
of $\mathscr{L}_{f}^{i} h(x)$ where $x_{1}$ is a factor. We get

$$
\begin{aligned}
\mathrm{d} \mathscr{L}_{f}^{0} h(x)^{T} & =\left(\begin{array}{l}
1 \\
0 \\
0
\end{array}\right), \quad \mathrm{d} \mathscr{L}_{f}^{1} h(x)^{T}=\left(\begin{array}{c}
x_{3}-x_{2} \\
-x_{1} \\
x_{1}
\end{array}\right) \\
\mathrm{d} \mathscr{L}_{f}^{2} h(x)^{T} & =\left(\begin{array}{c}
\left(x_{3}-x_{2}\right)^{2}-3 x_{1}^{2}+x_{2} \\
x_{1}\left(1-2\left(x_{3}-x_{2}\right)\right) \\
2 x_{1}\left(x_{3}-x_{2}\right)
\end{array}\right) .
\end{aligned}
$$

It is obvious that $\operatorname{dim} \mathrm{d} \mathscr{O}(x)=3$ on any connected subset of $\mathscr{S}=\left\{x \in \mathbb{R}^{3}:\left|x_{1}\right|>\varepsilon\right\}$, for any $\varepsilon>0$. However there is a singularity of the system observability for $x_{s}=\left(\begin{array}{lll}0 & x_{2} & x_{3}\end{array}\right)^{T}$ since $\operatorname{dim} \mathfrak{d} \mathscr{O}\left(x_{s}\right)=1$.

Physically, this singularity is related to the fact that one can not access to any information about state $x_{2}$ or $x_{3}$ when $x_{1}$ is null. This event rarely occurs on an uncontrolled system; yet, since the control objective will aim at stabilizing the system around $x^{*}$, the study and the avoidance of the observability singularity become decisive for the flow estimation.

\section{NONSINGULAR OBSERVER SYNTHESIS}

The core idea of this observer relies on the works [21][22]. Trying to exploit nonlinear observability normal forms may require a change of coordinates, generically an immersion from natural coordinates $x$ to target coordinates $z$. The main idea is to remove this observability singularity using both an extension and a coordinate space dimension augmentation. The former is the extension of this mapping for its inverse to be surjective, using fictitious outputs that make it possible to get (fake) information about the full state even at the system singularity. The latter is the augmentation of the (natural) coordinates using a dynamic extension to get a diffeomorphism between the natural state space and the target space.

The standard high gain observer [23], [24], written in the natural coordinates $x$, is first presented to better understand the way the singularity can be avoided.

\section{Theorem 3 (Standard High Gain Observer) Let $z=\phi(x)$} with

$$
z_{i}=\mathscr{L}_{f}^{i-1} h(x), i \leq 3
$$

A high gain observer for system (5) on either $\mathscr{S}^{-}=\{x \in$ $\left.\mathbb{R}^{3}: x_{1}<-\varepsilon\right\}$ or $\mathscr{S}^{+}=\left\{x \in \mathbb{R}^{3}: x_{1}>\varepsilon\right\}$, for any $\varepsilon>0$, is given by

$$
\dot{\hat{x}}=\frac{\partial \phi}{\partial x}(\hat{x})^{-1}(A \phi(\hat{x})+\operatorname{sat}(F)+\Delta K(y-C \hat{x})) .
$$

$A$ is the matrix whose only non null entries are a unitary superdiagonal, sat $(F)$ is a saturation function of $F$ whose non null entry is

$$
F_{3}(\hat{x})=\hat{x}_{1}\left[\left(\hat{x}_{3}-\hat{x}_{2}\right)^{3}+\left(\hat{x}_{3}-\hat{x}_{2}\right)\left(3 \hat{x}_{2}-5 \hat{x}_{1}^{2}\right)+\hat{x}_{1}^{2}-\hat{x}_{2}\right] .
$$

$\Delta$ is a diagonal matrix formed in ascending powers of a gain $\theta>\theta_{0}>1$ whose choice is related to the Lipschitz constant of $F . K$ is chosen to have the matrix $(A-K C)$ Hurwitz.
Proof: Using $z=\phi(x)$ with (10), it is straightforward that system (5) leads to

$$
\dot{z}=A z+F
$$

with $A$ and $F$ given in (11)-(12). A high gain observer synthesis for system (13) is given by

$$
\dot{\hat{z}}=A \hat{z}+\operatorname{sat}(F)+\Delta K C\left(y-\hat{z}_{1}\right) .
$$

A first traditional approach is to get $\hat{x}$ using the inversion of $\phi: \hat{x}=\phi^{-1}(\hat{z})$. However, this inversion can be tricky for a nonlinear mapping $\phi$. Another approach is to synthesize the observer in the natural coordinates $x$ provided that $\phi$ is a diffeomorphism

$$
\dot{\hat{x}}=\frac{\partial \phi}{\partial x}(\hat{x})^{-1} \dot{\hat{z}}
$$

In our study, it is obvious that $\phi$ is a diffeomorphism only on $\mathscr{S}^{-}$or on $\mathscr{S}^{+}$due to the observability singularity.

Remark 1 This standard high gain observer is not defined at the observability singularity since $\phi$ is by construction singular for $\hat{x}_{1}=0$.

$$
\frac{\partial \phi}{\partial x}(x)=\left(\begin{array}{ccc}
1 & 0 & 0 \\
x_{3}-x_{2} & -x_{1} & x_{1} \\
q_{2} & x_{1}\left(1-2 q_{1}\right) & 2 x_{1} q_{1}
\end{array}\right)
$$

with

$$
q_{1}=x_{3}-x_{2}, \text { and } q_{2}=q_{1}^{2}-3 x_{1}^{2}+x_{2} .
$$

The observability singularity at $x_{1}=0$ stated in Theorem 2 means that there is no access to any information about states $x_{2}$ and $x_{3}$ when $x_{1}=0$. So a way to provide such information when $x_{1}$ is close to the singularity is to add fake outputs, allowing for an access to the last two states in such a case. However, completing the $z$ state by the fake output makes the cardinality of this extended state space greater than the cardinality of the $x$ state space. It follows that the mapping $\phi$ is no more surjective. We thus propose to extend $\phi$ into a diffeomorphism $\Phi$ using a dynamic extension and a jacobian matrix completion.

Theorem 4 (Observer with Singularity Avoidance) Let

$$
\rho: x \mapsto \max \left(0, \varepsilon^{2}-x_{1}^{2}\right)^{2}
$$

for some $\varepsilon>0$. Consider the fake output

$$
y_{f}(x)=\left(\begin{array}{l}
\rho(x) x_{2} \\
\rho(x) x_{3}
\end{array}\right),
$$

and under the assumptions and notations of Theorem 3, we define two new state vectors $\bar{x}$ and $\bar{z}$ such as

$$
\bar{x}=\left(\begin{array}{l}
x \\
\tau
\end{array}\right) \text { and } \bar{z}=\Phi(\bar{x})=\left(\begin{array}{c}
z \\
y_{f}
\end{array}\right)+\Psi(x) \tau
$$

where $\tau \in \mathscr{T}$ are the exogenous variables added to the original state vector, $\mathscr{T}$ is a bounded subset of $\mathbb{R}^{2}$ and

$$
\Psi: x \mapsto\left(\begin{array}{cc}
0 & 0 \\
\rho(x) & 0 \\
0 & -\rho(x) \\
x_{1} & x_{1} \\
0 & x_{1}
\end{array}\right)
$$


Then a high gain observer for system (5) on any bounded subset $\mathscr{X} \in \mathbb{R}^{3}$ avoiding the singularity is given by

$$
\dot{\bar{x}}=\frac{\partial \Phi}{\partial \bar{x}}(\hat{\bar{x}})^{-1} \varphi(\hat{\bar{x}}, y)
$$

where

$$
\varphi:(\hat{\bar{x}}, y) \mapsto\left(\begin{array}{c}
A \Phi(\hat{\bar{x}})+\operatorname{sat}(F)+\Delta K(y-C \hat{\bar{x}}) \\
-k_{4} \Phi_{4}(\hat{\bar{x}}) \\
-k_{5} \Phi_{5}(\hat{\bar{x}})
\end{array}\right)
$$

with $\Phi_{i}$ denoting the $i$-th component of $\Phi$ given in (20), $k_{4}, k_{5}>0$ and A, $K, \Delta$ given by Theorem (3).

Proof: To circumvent the observability singularity, we modify the system outside $\mathscr{S}=\left\{x \in \mathbb{R}^{3}:\left|x_{1}\right|>\varepsilon\right\}$ by adding the fake output $y_{f}$ given by (19). It is worth noticing that this fake output is null on $\mathscr{S}$ so the system is not affected when living on $\mathscr{S}$. We now define a new mapping $\phi_{f}: x \mapsto \bar{z}=$ $\left(z, y_{f}\right)$, whose jacobian matrix is

$$
\frac{\partial \phi_{f}}{\partial x}(x)=\left(\begin{array}{cc}
1 & 0_{1,2} \\
J_{1}(x) & J_{2}(x)
\end{array}\right)
$$

with $0_{n, m}$ denoting the null matrix having $n \times m$ entries and matrices

$$
J_{1}(x)=\left(\begin{array}{c}
x_{3}-x_{2} \\
q_{2} \\
\frac{\partial \rho}{\partial x_{1}} x_{2} \\
\frac{\partial \rho}{\partial x_{1}} x_{3}
\end{array}\right), J_{2}(x)=\left(\begin{array}{cc}
-x_{1} & x_{1} \\
x_{1}-2 x_{1} q_{1} & 2 x_{1} q_{1} \\
\rho & 0 \\
0 & \rho
\end{array}\right) .
$$

The jacobian matrix of $\phi_{f}$ is thus full rank provided that $J_{2}$ is also full rank. The determinant of the first two lines of $J_{2}$ is $\left(-x_{1}^{2}\right)$ whilst the determinant of its last two lines is $\rho^{2}$. By definition (18), $\rho$ and $x_{1}$ can not be simultaneously null and it follows that $J_{2}(x)$ is full rank on $\mathbb{R}^{3}$. The fake outputs thus enable to get around the observability singularity.

Since the mapping $\phi_{f}$ is an immersion from $\mathbb{R}^{3}$ in $\mathbb{R}^{5}$, a way to get a one-to-one mapping is to extend it into a diffeomorphism $\Phi$. To do so, we propose a dynamic extension by augmenting the original state $x$ with $\tau=\left[\tau_{1} \tau_{2}\right]^{T}$ as given by (20)-(21). The resulting jacobian matrix of $\Phi$ is

$$
\frac{\partial \Phi}{\partial \bar{x}}(\bar{x})=\left(\begin{array}{cc}
1 & 0_{1,4} \\
\bar{J}_{1}(\bar{x}) & \bar{J}_{2}(\bar{x})
\end{array}\right)
$$

where the matrices $\bar{J}_{1}(\bar{x})$ and $\bar{J}_{2}(\bar{x})$, completed by the $\Psi$ columns (see (21)), and $\bar{J}_{1}(\bar{x})$ are given by

$$
\begin{aligned}
\bar{J}_{1}(\bar{x})= & J_{1}(\bar{x})+\left(\begin{array}{c}
\frac{\partial \rho}{\partial \bar{x}_{1}} \tau_{1} \\
-\frac{\partial \rho}{\partial \bar{x}_{1}} \tau_{2} \\
\tau_{1}+\tau_{2} \\
\tau_{2}
\end{array}\right), \\
\bar{J}_{2}(\bar{x})= & \left(\begin{array}{cccc}
-\bar{x}_{1} & \bar{x}_{1} & \rho & 0 \\
\bar{x}_{1}-2 \bar{x}_{1} q_{1} & 2 \bar{x}_{1} q_{1} & 0 & -\rho \\
\rho & 0 & \bar{x}_{1} & \bar{x}_{1} \\
0 & \rho & 0 & \bar{x}_{1}
\end{array}\right) .
\end{aligned}
$$

It is straightforward that $\operatorname{det} \bar{J}_{2}(\bar{x})=-\left(\rho^{2}+\bar{x}_{1}^{2}\right)^{2}<0$, so $\frac{\partial \Phi}{\partial \bar{x}}$ is invertible on any bounded subset of $\mathbb{R}^{3}$, and the observer (22) is thus well defined on any bounded subset of $\mathbb{R}^{5}$.
To ensure that we have

$$
\lim _{t \rightarrow \infty}\|x(t)-\hat{x}(t)\|+\|\hat{\tau}(t)\|=0
$$

along the solutions $(x, \hat{x}, \hat{\tau})(t)$ of (5) and (22)-(23), one can notice that the inverse mapping $\Phi^{-1}: \bar{z} \mapsto \bar{x}$ is given by

$$
\Phi^{-1}(\bar{z})=\left(\begin{array}{c}
\bar{z}_{1} \\
\frac{-\bar{z}_{1}^{2} \alpha^{2}-\bar{z}_{1} \rho \beta\left(\bar{z}_{1}^{2}+\rho^{2}\right)+\gamma\left(\bar{z}_{1}^{2}+\rho^{2}\right)^{2}}{\left(\bar{z}_{1}^{2}+\rho^{2}\right)^{3}} \\
\frac{-\bar{z}_{1}^{2} \alpha^{2}-\bar{z}_{1} \rho \beta\left(\bar{z}_{1}^{2}+\rho^{2}\right)+(\gamma+\alpha)\left(\bar{z}_{1}^{2}+\rho^{2}\right)^{2}}{\left(\bar{z}_{1}^{2}+\rho^{2}\right)^{3}} \\
\frac{\beta}{\bar{z}_{1}^{2}+\rho^{2}} \\
\frac{\bar{z}_{1} \rho \alpha\left(\alpha-\left(\bar{z}_{1}^{2}+\rho^{2}\right)\right)}{\left(\bar{z}_{1}^{2}+\rho^{2}\right)^{3}}+\frac{\delta}{\bar{z}_{1}^{2}+\rho^{2}}
\end{array}\right)
$$

with

$$
\begin{aligned}
& \alpha(\bar{z})=\bar{z}_{1} \bar{z}_{2}+\rho\left(\bar{z}_{5}-\bar{z}_{4}\right) \\
& \beta(\bar{z})=\rho \bar{z}_{2}-\bar{z}_{1}\left(\bar{z}_{5}-\bar{z}_{4}\right) \\
& \gamma(\bar{z})=\bar{z}_{1} \bar{z}_{3}+\bar{z}_{1}^{4}+\rho \bar{z}_{4} \\
& \delta(\bar{z})=\bar{z}_{1} \bar{z}_{5}-\rho\left(\bar{z}_{1}^{3}+\bar{z}_{3}\right) .
\end{aligned}
$$

So $\Phi^{-1} \in \mathscr{C}^{1}\left(\mathbb{R}^{5}\right)$ and it follows that it is a $L$-Lipschitz mapping on any bounded subset of $\mathbb{R}^{5}$. Besides, from (20)(21), we have $\Phi(x, 0)=\phi_{f}(x)$, so it is straightforward that for $\bar{z}=\Phi(x, 0)$ :

$$
\begin{aligned}
& \left\|\Phi^{-1}(\bar{z})-\Phi^{-1}(\hat{\bar{z}})\right\| \leq L\|\bar{z}-\hat{\bar{z}}\| \\
& \left\|\left(\begin{array}{c}
x \\
0_{2,1}
\end{array}\right)-\left(\begin{array}{c}
\hat{x} \\
\hat{\tau}
\end{array}\right)\right\| \leq L\left\|\phi_{f}(x)-\Phi(\hat{x}, \hat{\tau})\right\| .
\end{aligned}
$$

Since we have proven in Theorem 3 that the first three lines of (23) is a converging observer of (13), it follows from (31) that (28) holds. The dynamics of the last two states $\bar{z}$ in (23) are arbitrarily set by any strictly positive gains $k_{4}$ and $k_{5}$.

\section{SIMULATIONS AND EXPERIMENTS}

\section{A. Simulation results}

Simulations are performed with the high gain observer (HGO) proposed in Theorem 4. The $x_{1}$ dynamics and the output $y$ are corrupted by the addition of zero mean Gaussian white noises of standard deviations 0.4 and 0.1 , respectively. This additional noise on the dynamics is used to model the influence of other modes on the $x_{1}$ behaviour. During the time interval 10-15 seconds, a state feedback is applied to stabilize the state $x_{1}$ to zero, thus forcing the system to be singular. The different parameters (initial values and observer gain $\left.K_{H G O}=\left(K^{T} k_{4} k_{5}\right)^{T}\right)$ are given in Table I.

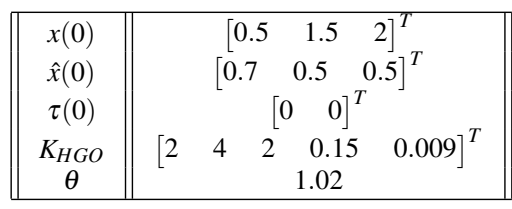

TABLE I

INITIAL CONDITIONS AND OBSERVER PARAMETERS

The value of the high gain $\theta$ is chosen quite small in order to avoid sensitivity issues (in particular with respect 


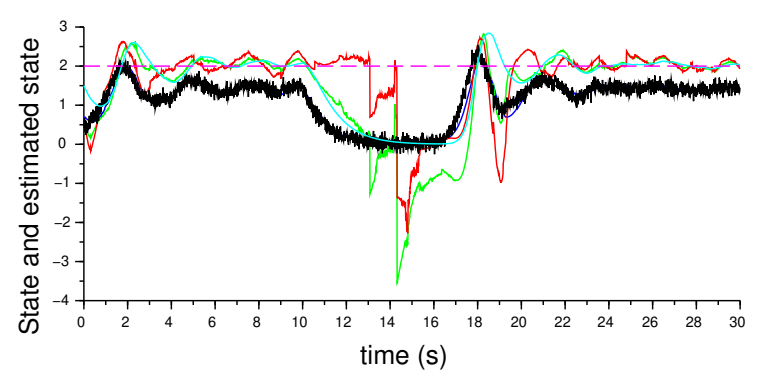

Fig. 1. High gain observer : the system states $x_{1}, x_{2}, x_{3}$ are depicted in black, cyan and purple, the estimations $\hat{x}_{1}, \hat{x}_{2}, \hat{x}_{3}$ are depicted in blue, green and red lines, respectively.

to the output noise), to lessen the peaking effect and make the system performance effective (Fig 1) despite noise on the state dynamics. When the state feedback stabilizes $x_{1}$ to zero for $t \in[10,15] s$, the singularity is avoided using the fake outputs to preserve the mapping $\Phi$ invertibility. The choice of a quite small $\varepsilon=0.001$ for (18) allows to modify the system only for a short period, yet at the price of the convergence of the estimated growth rate to zero instead of the nominal value $x_{3}=2$.

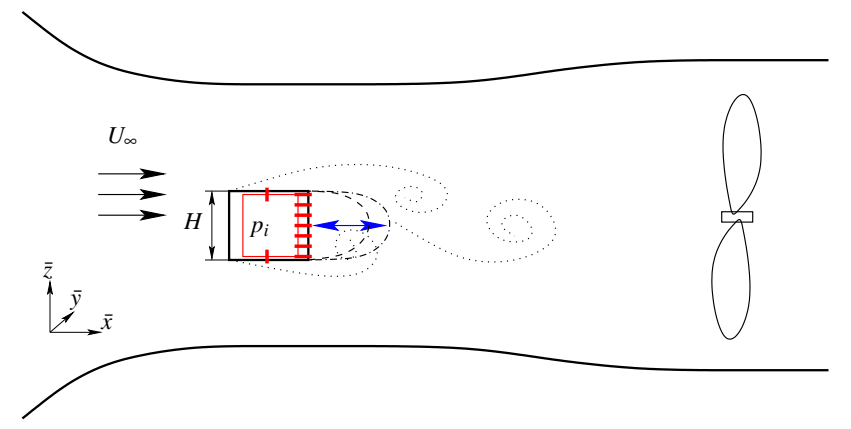

Fig. 2. Sketch of the open wind tunnel and the square cylinder experiment. The thick red lines show the pressure taps while the pressure transducer is located inside the bluff body. The double arrow in blue shows the variation of the shift mode while the vortex shedding is shown with dotted line.

\section{B. Experimental Setup}

Experiments were led in the $40 \times 40 \mathrm{~cm}^{2}$ test section of an open wind tunnel. Speeds $U_{\infty}$ ranging from 5 to $10 \mathrm{~m} / \mathrm{s}$ were used to generate the flow around a square cylinder of length $H=0.1 \mathrm{~m}$. The cylinder was equipped with 9 pressure taps located on the sides and on the back of the object, shown in Fig. 2. Here confinement is not an issue but it should be noted that the blockage ratio is $1 / 4$ in the test section which has an influence on the characteristics of the vortex shedding compared to the unconfined flow. The Reynolds number $R e=U_{\infty} H / v$, where $v=1.810^{-5}$ $\mathrm{m}^{2} / \mathrm{s}$ is the kinematic viscosity of the air, was in the range of $[2.7 ; 5.5] \times 10^{4}$. A second non-dimensional number of importance is the frequency of the vortex shedding $f=$ $S t U_{\infty} / H$, where the Strouhal number $S t=0.14$ in the case of the square cylinder. The side pressure sensors were used to measure the amplitude of vortex shedding while seven pressure sensors positioned on the back of the cylinder were used to measure the pressure component of the drag force onto the bluff body. The variables are nondimensionalized as follows:

$$
\overline{\mathbf{x}}=\overline{\mathbf{x}}^{*} / H, \mathbf{u}=\mathbf{u}^{*} / U_{\infty}, \text { and } p=2 p^{*} /\left(\rho U_{\infty}^{2}\right),
$$

where the starred quantities are the dimensional quantities. Pressures were considered as the pressure coefficient $C_{p}=$ $2\left(p-p_{\infty}\right) /\left(\rho U_{\infty}^{2}\right)$ measured at each position on the cylinder. The side faces contribute only to the lift coefficient $C_{L}$ whereas the drag coefficient $C_{D}$ corresponds to the projection of the pressure coefficient onto the front and back surfaces. Total drag is defined as the sum of pressure and friction drags: $C_{D}=C_{p}+C_{f}$. Numerical simulations and experimental measurements report mean values of $C_{D} \approx 2.2$ [25], [26] from Reynolds Averaged Navier-Stokes (RANS) simulations and $C_{D} \approx 2.19$ [27] from Direct Numerical Simulations (DNS) and mean $C_{L}=0$ for very similar Reynolds numbers. In the present experiment, these mean values are found to be $\left[C_{D, \text { exp }}, C_{L, \text { exp }}\right] \approx[1.97,0.05]$ which is in good agreement, the former being necessarily different because of the contribution of the viscous drag which may account for the $10 \%$ difference between total and pressure drag (i.e. $C_{f} \approx 0.22$ ). In addition, [25] and [26] found using RANS simulations that the drag coefficient of the steady state is $C_{D, \text { steady }} \approx 1.71$ for a similar Reynolds number, which is directly related to the shift mode $x_{2}$, that is $\Delta C_{D, \exp }(t)=C_{D, \exp }(t)-C_{D, \text { steady }} \sim x_{2}$.

Fluctuations can also be used to compare DNS results [27] with the present experiments. In particular, the fluctuations of the lift coefficient provide another mean to compare the amplitude of the oscillations from the pressure taps located on the sides of the square cylinder where [27] report $r m s\left(C_{L}(t)\right) \approx 1.71$. This coefficient can only be inferred from two pressure taps (see Fig. 2) and provide an estimation $\operatorname{rms}\left(C_{L, \exp }(t)\right) \approx 0.54$ which is well below the value found in the literature. This difference may be attributed to the sparse measurements on these faces of the square cylinder.

In the case of a bluff body, drag is more conveniently controlled by modifying the size of the recirculation region, which in turn, is slaved to the amplitude of the vortex shedding, at least in the laminar case. It is however less clear that the dominant vortex shedding mode alone is enough to predict the modification of the shift mode in the turbulent case and the prediction of the pressure at the base from a minimum number of sensors remains an open question. Our aim is to relate the amplitude of the shift mode through the amplitude of the vortex shedding which can be evaluated using sensors located on both sides of the bluff body. As shown in [11], [12], the velocity on opposite sides of the bluff body is shifted by a phase of $\pi / 2$. However, using pressure measurements $\left(p_{1}, p_{2}\right)$, the signal obtained from similar locations are shifted with a phase of $\pi$ [28]. To overcome this problem, we define $\bar{p}_{1}=\left(p_{1}-p_{2}\right) / 2$ and $\bar{p}_{2}$ the temporal derivative of the former, $\bar{p}_{2}=\partial_{t}\left(p_{1}-p_{2}\right)$.

The amplitude of the vortex shedding can be written as:

$$
A_{m p}(t)=\sqrt{\bar{p}_{1}^{2}(t)+\bar{p}_{2}^{2}(t)},
$$


(a)

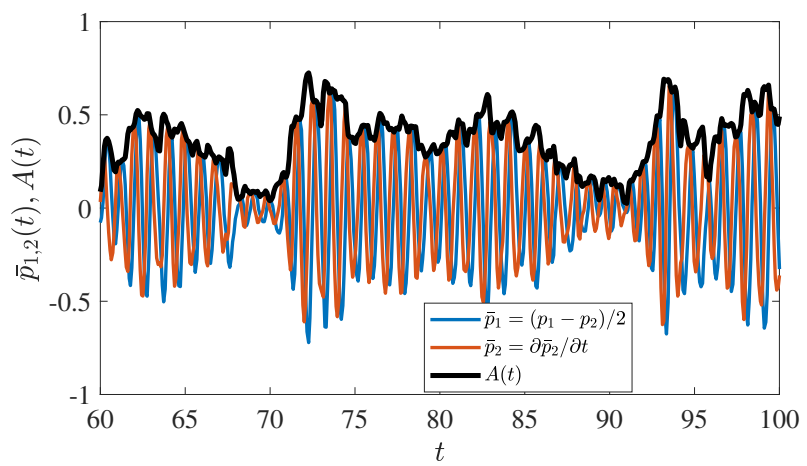

(b)

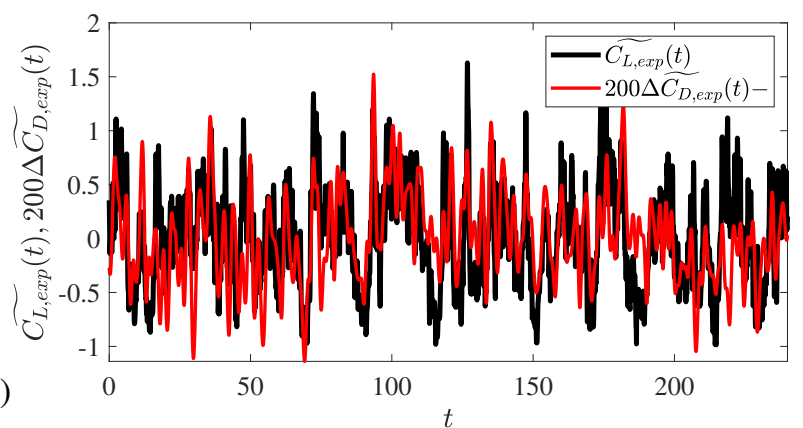

(c)

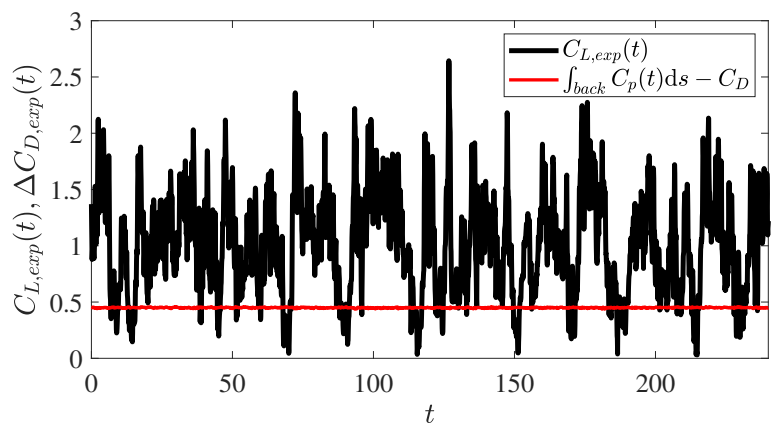

Fig. 3. (a) Temporal evolution of the mean pressure $\bar{p}=\left(p_{1}-p_{2}\right)$ (blue) where $p_{1}$ and $p_{2}$ are the pressures measured on the sides of the square cylinder respectively, parallel to the flow and the temporal derivative (red). (b) Estimated lift coefficient $\widetilde{C}_{L, 2 x p}(t)$ (black) compared with fluctuations of the pressure on the back $\Delta \widetilde{C}_{D, \exp }(t)$ magnified by a factor 200 . The $(\sim)$ denotes that the mean was subtracted. (c) Temporal evolution of the lift coefficient $C_{L, \exp }$ and the shift of the drag coefficient $\Delta C_{D, \exp }(t)$ with respect to the steady state.

illustrated in Fig. 3(a). Fig. 3(c) shows that the mean pressure at the base and thus the drag coefficient deviation from the steady state $\Delta C_{D, \exp }(t)$ is essentially insensitive to the intermittency of the vortex shedding amplitude. The small oscillation of $\Delta C_{D, \text { exp }}(t)$ are magnified in 3(b) together with a low-pass filter computed over 20 periods of vortex shedding where the slow dynamics appear to be well correlated with the variations of the amplitude $A_{m p}$. As proposed by [29] a brief time-scale analysis shows that the shear-time scale $t_{s} \sim$ $H / S t U_{\infty} \sim \mathscr{O}\left(10^{-1}\right)$ akin to vortex shedding is much shorter than the nonlinear time scale $t_{n l} \sim H / S t u^{\prime} \sim \mathscr{O}\left(10^{0}\right)$ where $u^{\prime}$ is the amplitude of the turbulent fluctuations, typically of the order $\mathscr{O}\left(U_{\infty} / 10\right)$. This nonlinear time scale is visible for

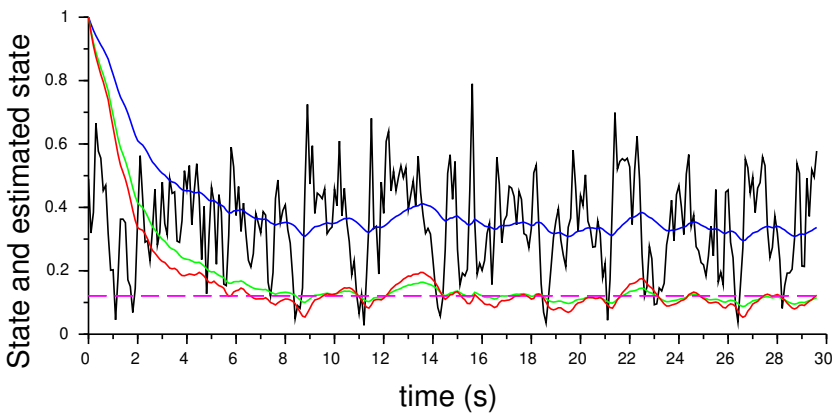

Fig. 4. Experimental estimated states. The black solid line represents the measured output $y=x_{1}$ on the system, the purple dotted line is the theoretical shift mode and growth ratio at the equilibrium, computed as $\langle y\rangle^{2}$. Estimated states $\hat{x}_{1}, \hat{x}_{2}$ and $\hat{x}_{3}$ are depicted by blue, green and red solid lines respectively.

instance in Fig. 3 and Fig. 4 and modulates the amplitude of the vortex shedding in time, resulting in the intermittent amplitude $A_{m p}(t)$.

\section{Experimental results}

We apply the high gain observer proposed in Theorem 4 to the experimental data. Since we have no access to the shift mode nor to the growth rate, we provide theoretical values based on the equilibrium $x^{* * *}:\langle y\rangle^{2}=0.1225$. The observer is initialized with $\hat{x}(0)=\left(\begin{array}{lll}1 & 1 & 1\end{array}\right)^{T}$ and $\tau(0)=0_{2,1}$. Gains are chosen as $K=\left(\begin{array}{lll}0.31 & 0.07 & 0.05\end{array}\right)^{T}$ and the high gain as $\theta=1.1$ to filter the signal high frequencies. After a $6 s$ long transient phase, the observer converges both to the measured amplitude and to the theoretical values of the shift mode and growth rate (Fig. 4). The filtering effect of the observer drastically reduces the noise impact on the estimated states. Statistical analysis is given in Table II. Despite a very simple model, it seems that the proposed approach is able to catch the dynamics of the mode. However, since many modes are implied for this large Reynolds number, it will be of great interest to compare the theoretical and estimated values to their true values, and to estimate the contribution of the other modes dynamics.

\begin{tabular}{||l||l||l||}
\hline Variable & Mean value & Standard deviation \\
\hline$y=x_{1}$ & 0.03479 & 0.1486 \\
$\hat{x}_{1}$ & 0.3430 & 0.0377 \\
$\hat{x}_{2}$ & 0.1191 & 0.0255 \\
$\hat{x}_{3}$ & 0.1138 & 0.0498 \\
\hline
\end{tabular}

TABLE II

STATISTICAL VALUES FOR EXPERIMENTS

\section{CONCLUSIONS AND FUTURE WORKS}

This study provides the necessary grounds to verify whether nonlinear observers based on empirical Galerkin model can capture the dynamics of the vortex shedding and shift mode at high Reynolds number. The experimental results using the proposed high gain observer are very 
encouraging for handling the observability singularity, an issue of great interest for active control since the control objective is to stabilize the amplitude to zero.

On-going works are twofold. We are first interested in observation of such a system for a higher number of modes, where the high gain standard approaches can suffer from an increased sensitivity to noise as the state dimension increases, the same goes for the power of the high gain. A promising tool to extend the present approach is the recently high gain observer with limited gain powers [30]. Another perspective is the synthesis of output feedbacks using the estimated state to bring the system to a symmetric wake flow which is a promising way to minimize the energy consumption of bluff bodies.

\section{ACKNOWLEDGMENTS}

The authors gratefully acknowledge the financial support from the Region Centre Val de Loire (France) under the project APR-IA COMODO.

\section{REFERENCES}

[1] G. Sovran, Aerodynamic drag mechanisms of bluff bodies and road vehicles. Springer Science \& Business Media, 2012.

[2] K. R. Cooper, "Bluff-body aerodynamics as applied to vehicles," $J$. Wind Eng. Indus. Aero., vol. 49, no. 1-3, pp. 1-21, 1993.

[3] X. Ma, G.-S. Karamanos, and G. Karniadakis, "Dynamics and lowdimensionality of a turbulent near wake," Journal of fluid mechanics, vol. 410, pp. 29-65, 2000.

[4] R. Volpe, P. Devinant, and A. Kourta, "Experimental characterization of the unsteady natural wake of the full-scale square back ahmed body: flow bi-stability and spectral analysis," Experiments in Fluids, vol. 56, no. 5, p. 99, 2015.

[5] A. Thacker, S. Aubrun, A. Leroy, and P. Devinant, "Experimental characterization of flow unsteadiness in the centerline plane of an ahmed body rear slant," Exp. Fluids, vol. 54, no. 3, p. 1479, 2013.

[6] J. Östh, B. R. Noack, S. Krajnović, D. Barros, and J. Borée, "On the need for a nonlinear subscale turbulence term in pod models as exemplified for a high-reynolds-number flow over an ahmed body," $J$. Fluid Mech., vol. 747, pp. 518-544, 2014.

[7] D. Sipp, O. Marquet, P. Meliga, and A. Barbagallo, "Dynamics and control of global instabilities in open-flows: a linearized approach," App. Mech. Rev., vol. 63, no. 3, p. 030801, 2010.

[8] C. W. Rowley and S. T. M. Dawson, "Model reduction for flow analysis and control," Ann. Rev. Fluid Mech., vol. 49, pp. 387-417, 2017.

[9] S. L. Brunton, J. L. Proctor, and J. N. Kutz, "Discovering governing equations from data by sparse identification of nonlinear dynamical systems," Proc. Nat. Acad. Sci., vol. 113, no. 15, pp. 3932-3937, 2016.

[10] J.-C. Loiseau and S. L. Brunton, "Constrained sparse galerkin regression," J. Fluid Mech., vol. 838, pp. 42-67, 2018.

[11] J. T. Stuart, "On the non-linear mechanics of hydrodynamic stability," J. Fluid Mech., vol. 4, no. 1, pp. 1-21, 1958.

[12] A. E. Deane, I. G. Kevrekidis, G. E. Karniadakis, and S. A. Orszag, "Low-dimensional models for complex geometry flows: application to grooved channels and circular cylinders," Phys. Fluids A: Fluid Dyn., vol. 3, no. 10, pp. 2337-2354, 1991.

[13] B. R. Noack, K. Afanasiev, M. Morzyński, G. Tadmor, and F. Thiele, "A hierarchy of low-dimensional models for the transient and posttransient cylinder wake," J. Fluid Mech., vol. 497, pp. 335-363, 2003.

[14] V. Mantič-Lugo, C. Arratia, and F. Gallaire, "Self-consistent mean flow description of the nonlinear saturation of the vortex shedding in the cylinder wake," Phys. Rev. Lett., vol. 113, no. 8, p. 084501, 2014.

[15] P.-Y. Passaggia and U. Ehrenstein, "Optimal control of a separated boundary-layer flow over a bump," J. Fluid Mech., vol. 840, pp. 238265, 2018.

[16] G. Berkooz, P. Holmes, and J. L. Lumley, "The proper orthogonal decomposition in the analysis of turbulent flows," Annual review of fluid mechanics, vol. 25, no. 1, pp. 539-575, 1993.
[17] C. W. Rowley, I. Mezić, S. Bagheri, P. Schlatter, and D. S. Henningson, "Spectral analysis of nonlinear flows," J. Fluid Mech., vol. 641, pp. $115-127,2009$.

[18] P. J. Schmid, "Dynamic mode decomposition of numerical and experimental data," J. Fluid Mech., vol. 656, pp. 5-28, 2010.

[19] A. Wynn, D. Pearson, B. Ganapathisubramani, and P. J. Goulart, "Optimal mode decomposition for unsteady flows," Journal of Fluid Mechanics, vol. 733, pp. 473-503, 2013.

[20] D. Sipp and P. J. Schmid, "Linear closed-loop control of fluid instabilities and noise-induced perturbations: a review of approaches and tools," Applied Mechanics Reviews, vol. 68, no. 2, p. 020801, 2016.

[21] V. Andrieu, J.-B. Eytard, and L. Praly, "Dynamic extension without inversion for observers," IEEE International Conference on Decision and Control, pp. 878 - 883, 2014.

[22] P. Bernard, V.Andrieu, and L. Praly, "Expressing an observer in preferred coordinates by transforming an injective immersion into a surjective diffeomorphism," SIAM J. Control Optim., vol. 56, no. 3, pp. 2327-2352, 2018.

[23] J. Gauthier, H. Hammouri, and S. Othman, "A simple observer for nonlinear systems. application to bioreactors." IEEE Transactions on Automatic Control, vol. 37, no. 6, pp. 875-880, 1992.

[24] J. Gauthier and I. Kupka, "Observability and observers for nonlinear systems," SIAM J. Control Optim., vol. 32, no. 4, pp. 975-994, 1994.

[25] G. Iaccarino, A. Ooi, P. Durbin, and M. Behnia, "Reynolds averaged simulation of unsteady separated flow," International Journal of Heat and Fluid Flow, vol. 24, no. 2, pp. 147-156, 2003.

[26] P. Meliga, O. Cadot, and E. Serre, "Experimental and theoretical sensitivity analysis of turbulent flow past a square cylinder," Flow, Turbulence and Combustion, vol. 97, no. 4, pp. 987-1015, 2016.

[27] F. Trias, A. Gorobets, and A. Oliva, "Turbulent flow around a square cylinder at reynolds number 22,000: A dns study," Computers \& Fluids, vol. 123, pp. 87-98, 2015.

[28] D. Kurtulus, F. Scarano, and L. David, "Unsteady aerodynamic forces estimation on a square cylinder by tr-piv," Exp. Fluids, vol. 42, no. 2, pp. 185-196, 2007.

[29] A. S. Sharma, J. F. Morrison, B. J. McKeon, D. J. N. Limebeer, W. H. Koberg, and S. J. Sherwin, "Relaminarisation of re $\tau=100$ channel flow with globally stabilising linear feedback control," Phys. Fluids, vol. 23 , no. 12, p. $125105,2011$.

[30] D. Astolfi and L. Marconi, "A high-gain nonlinear observer with limited gain power," IEEE Transactions on Automatic Control, vol. 60, no. 11, pp. 3059-3064, 2015. 